\title{
Experience Using the Heidelberg Retina Angiograph 2 with a Wide-Field Contact Lens System in Diabetic Retinopathy Cases
}

\author{
Miyo Shiibashi ${ }^{2}$, Miwako Yoshimoto ${ }^{1}$, Takashi Shigeeda ${ }^{2}$, Shigehiko Kitano ${ }^{3}$ and Satoshi Kato ${ }^{* 2}$ \\ ${ }^{1}$ Tokyo Teishin Hospital, Japan \\ ${ }^{2}$ Department of Ophthalmology and Visual Sciences, University of Tokyo, Japan \\ ${ }^{3}$ Tokyo Woman's Medical University Diabetic Center, Japan
}

\begin{abstract}
Introduction: HRA (Heiderberg Retina Angiograph) 2 uses a confocal scanning laser system which can provide high quality digital images but its imaging field is only $30^{\circ}$ at most. HRA2 with a wide-field contact lens system allows an imaging field of up to $150^{\circ}$.

Methods: We examined the advantages and disadvantages of HRA2, with a wide-field contact lens, for the evaluating diabetic retinopathy $(\mathrm{DR})$.

Results: HRA2 was beneficial for obtaining images of the entire retina simultaneously, without missing peripheral retinal non-perfusion and neovascularization. On the other hand, clear images connot be acquired in cases with media opacities such as corneal dystrophy, cataract and asteroid hyalosis, or in those with yellow tinted IOL.
\end{abstract}

Conclusions: HRA2 with a wide-field contact lens is useful for visualizing peripheral retinal lesions in DR cases.

Keywords: Heidelberg retina angiograph 2, wide-field contact lens, diabetic retinopathy.

\section{INTRODUCTION}

HRA2 uses a confocal scanning laser system and can provide high quality digital images. It can also simultaneously handle both digital fluorescein angiography (FA) and indocyanine green angiography (IA) [1]. It is beneficial for patients because the amount of light required for the examination is minimal, such that patients do not experience 'dazzling'. Enhanced contrast, details, and image sharpness are generated using confocality [2].

However, its imaging field is at most $30^{\circ}$ and it is commonly used for the diagnosis of macular diseases and retinal vascular occlusion in the posterior poll.

Wide-field fluorescein angiography using contact lenses has been reported to have numerous clinical applications to ensure high resolution and, high contrast [3].

Recently, it has become possible to cover an imaging field of up to 200 degree [4-6]. The utility of this technology in clinical practice continues to be investigated [6]. HRA2 with a wide-field contact lens provides up to a 150 degree field of view [4]. It is a valuable tool in the management of patients with posterior and peripheral uvetis, which may be difficult to detect clinically $[7,8]$.

In this report, we investigated the advantages and disadvantages of the evaluating diabetic retinopathy (DR) using HRA2 with a wide-field contact lens.

*Address correspondence to this author at the Department of Ophthalmology, University of Tokyo Graduate School of Medicine, 7-3-1, Hongo, Bunkyo-ku, Tokyo, 113-8655, Japan; Tel: +81-3-3815-5411, Ext. 33494; Fax: +81-3-3817-0798; E-mail: katou-s@ka2.so-net.ne.jp

\section{METHODS}

Fifty DR patients followed at Tokyo University Hospital or Tokyo Woman's Medical University Diabetic Center were enrolled. These patients underwent FA using both of the following instruments: the ordinary fundus camera and HRA2 which is a new model of HRA with a wide-field contact lens, the so-called "Staurenghi Lens".

HRA2 is a new model of HRA that has a higher resolution than HRA $(1,536 \times 1,536$ pixels vs $512 \times 512$ pixels). The HRA2 equipment is also smaller and it provides a higher resolution at a faster speed than HRA.

We subsequently compared the images obtained using these instruments with each other. For pictures taken with the ordinary camera, we made a composite image by assembling fundus photographs. This facilitated comparison with the HRA2 images. Furthermore the images obtained by HRA2 with the Staurenghi Lens are usually inverted, allowing us to change them into direct images.

With HRA2, we applied a mono-wavelength laser-beam of $488 \mathrm{~nm}$ as the an excition light for FA. The camera used in Tokyo University Hospital was the TRC $50 \mathrm{Ax}^{\circledR}$ (TOPCON) and that used in Tokyo Woman's Medical University Diabetic Center was the PR03 ${ }^{\circledR}$ (KOWA). We present cases in which HRA2 was helpful, and others in which it was not, for evaluating DR.

\section{RESULT}

First, we present cases in which HRA2 was useful. In these cases, there are non- perfusion areas or 
neovascularization at the far peripheral retina, and there is some possibility that these lesions will be missed with an ordinary camera, even after a careful search.

On the contrary, we were able to detect a lesion at one sight with HRA2 using the Staurenghi Lens (Figs. 1, 2). Moreover, the intensity of the leakage can be assessed using time course photography and more than one lesion can be seen at the same time with HRA2. It is impossible to do this with the composite image made using ordinary camera images in which each picture is taken at a different time point.

There were also cases in which HRA2 was not helpful. These cases had media opacities such as corneal dystrophy, cataract and asteroid hyalosis. The images obtained with HRA2 were not useful because fundus details were obscured by the media opacities. However, we were able to see the fundus clearly with the ordinary fundus camera (Figs. 3-5).

Many diabetic patients suffer from cataracts and we need to take this into consideration. HRA2 is weak in cases of media opacity especially cataracts.
Another case had an intraocular lens (IOL), making mydriasis inadequate. The peripheral fundus could not be visualized distinctly because of opacitiy at the anterior capsule edge (Fig. 6). The final case had a tinted IOL. In this case, the image obtained with HRA2 was slightly coarse as compared to those obtained with an ordinary camera (Fig. 7).

When using an ordinary fundus camera, the examiner's skill and the patients' cooperation in correcting eye position are essential for detecting lesions in the peripheral retina. Conversely, we can obtain images of the entire retina simultaneously without missing the peripheral area with HRA2. However, for cases with media opacities, these advantages of HRA2 are lost and the pictures obtained are inadequate as compared to those of an ordinary fundus camera.

\section{CONCLUSION}

Detection of a peripheral vessel leakage and of an untreated, peripheral, non-perfusion area is very important in the treatment of DR [9].
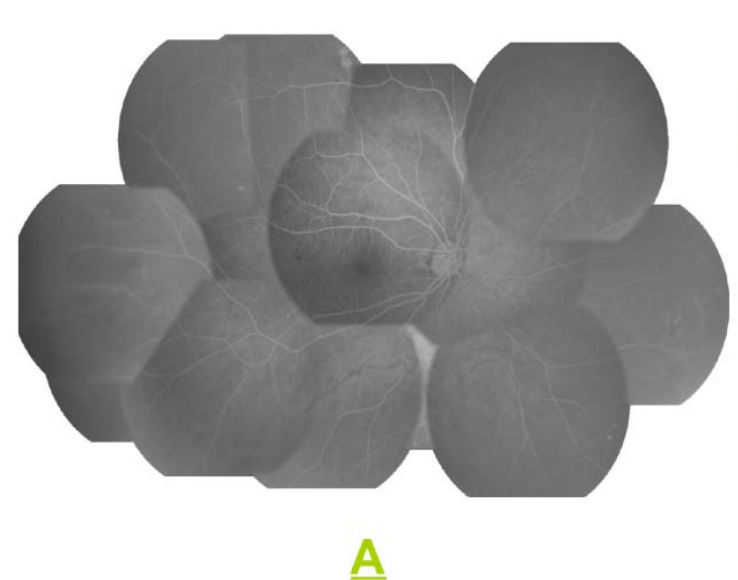

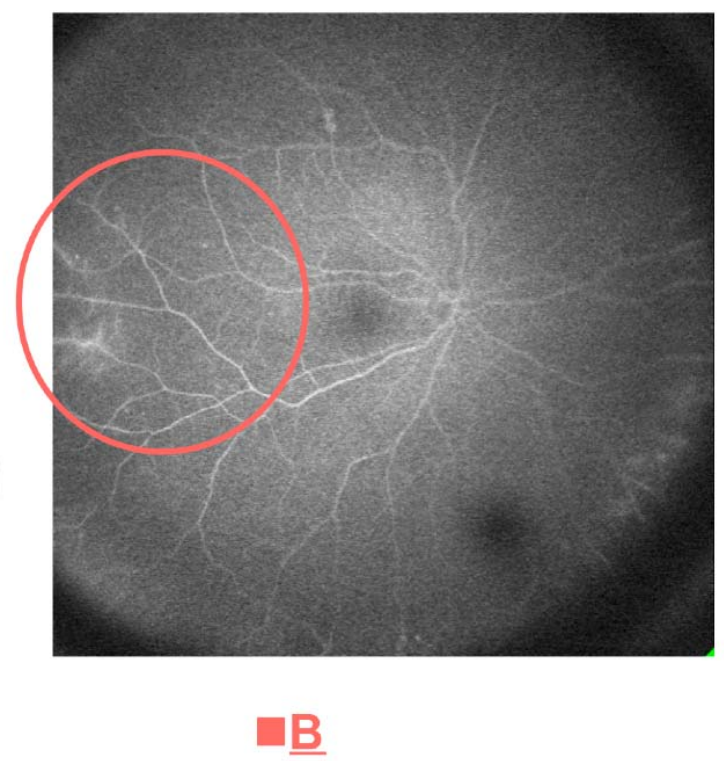

Fig. (1). Case with a peripheral non-perfusion area. A: Ordinary camera; B: HRA2.
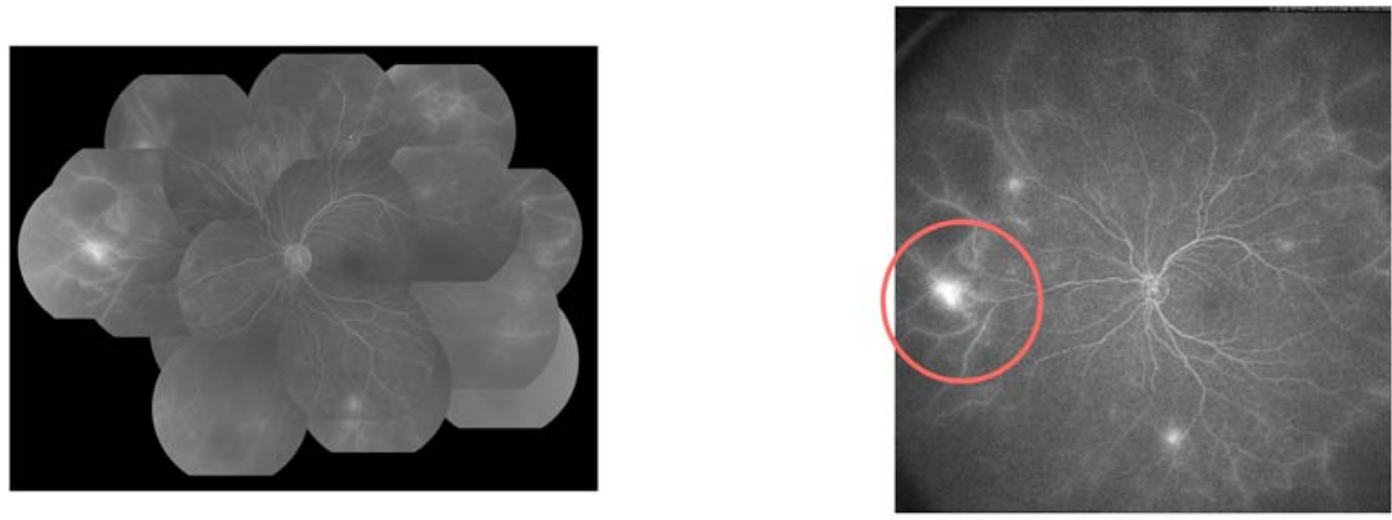

Fig. (2). Case with retinal neovascularization. A: Ordinary camera; B: HRA2. 

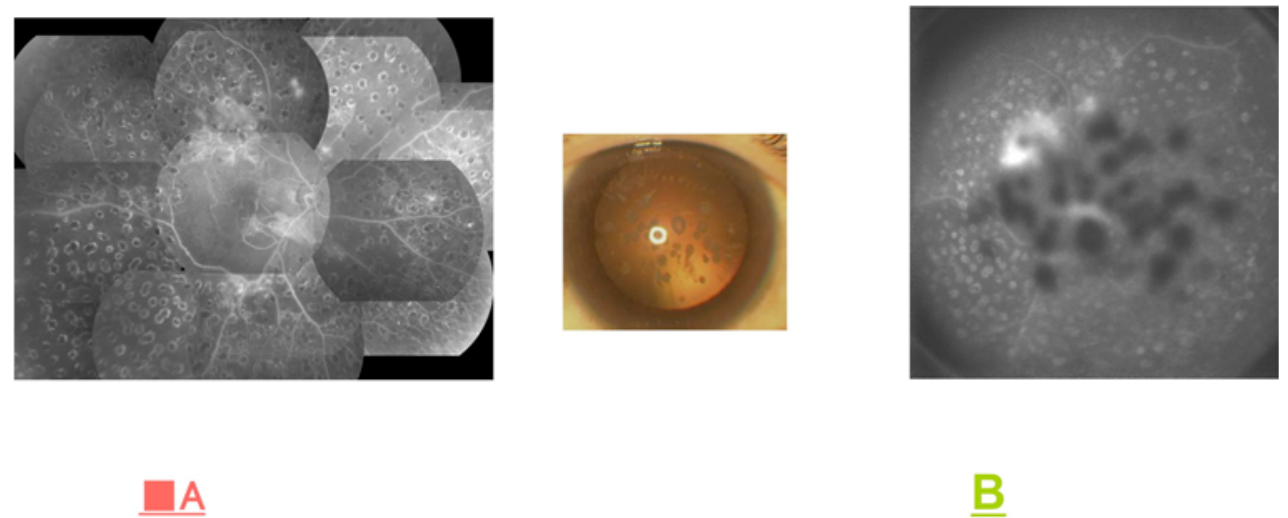

Fig. (3). Case with corneal opacities. A: Ordinary camera; B: HRA2.

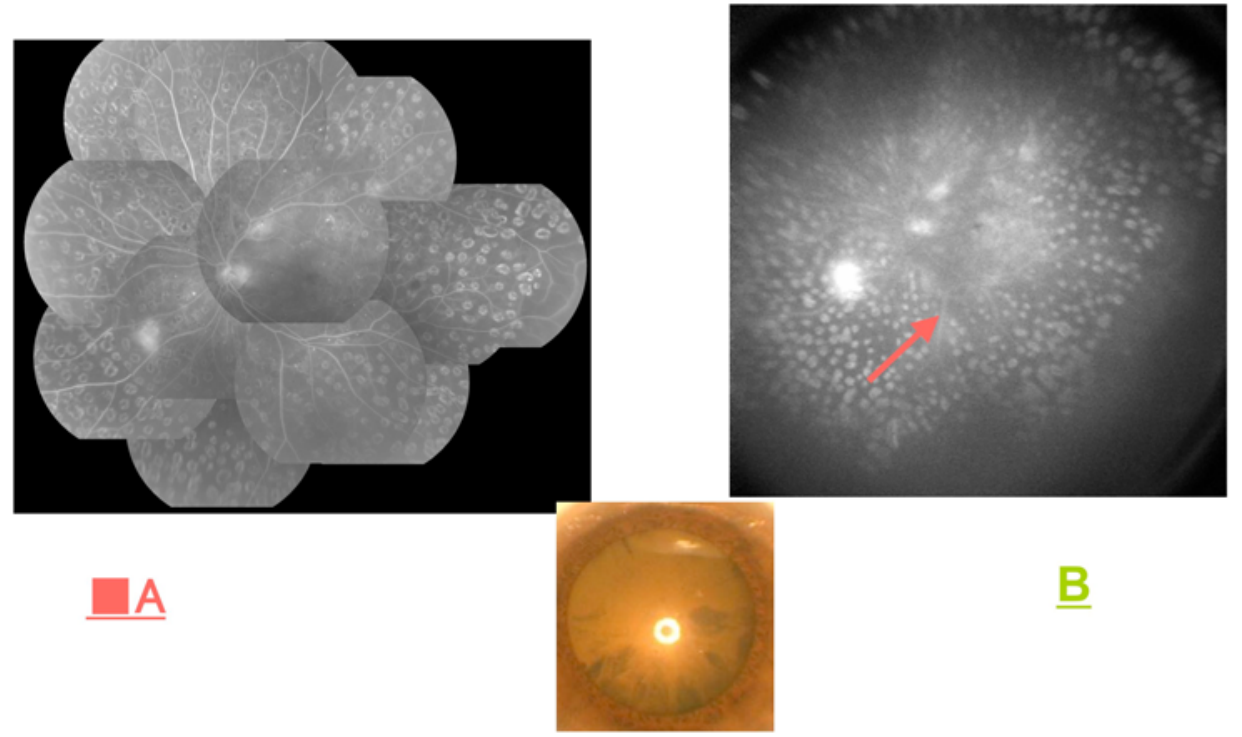

Fig. (4). Case with a nuclear cataract. No contrast is apparent. A: Ordinary camera; B: HRA2.
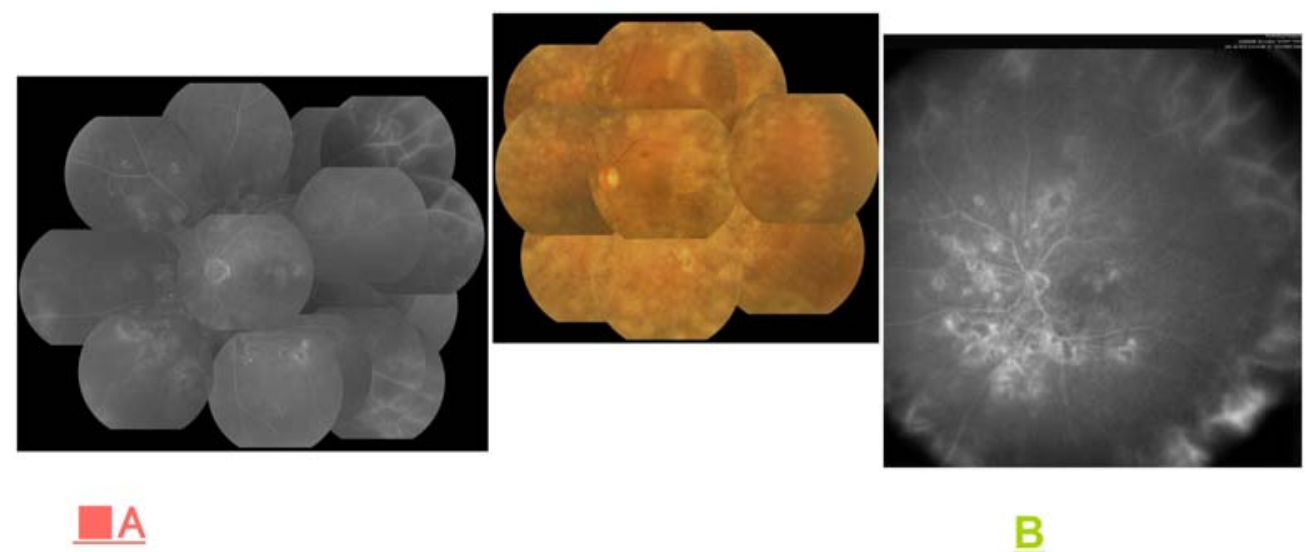

Fig. (5). Case with asteroid hyalosis. A: Ordinary camera; B: HRA2.

Wide-angle images up to 120 degrees can be easily produced in a few seconds by automatically combining multiple images. HRA2 is more useful than an ordinary camera when a patient cannot keep the correct eye position because of dazzling. HRA2 is thus beneficial for patients with incomplete eye movements.

Though HRA2 with a wide-field contact lens is useful for visualizing peripheral retinal lesions in DR cases, its applications are limited in cases with media opacities and yellow-tinted IOL.

We described a few distinctive cases as well as another 50 DR cases with little media opacity and a posterior pole retinal lesion; in the latter cases, there is not much difference between HRA2 and an ordinary fundus camera.

We should understand these advantages and disadvantages when examining DR patients using HRA2. 

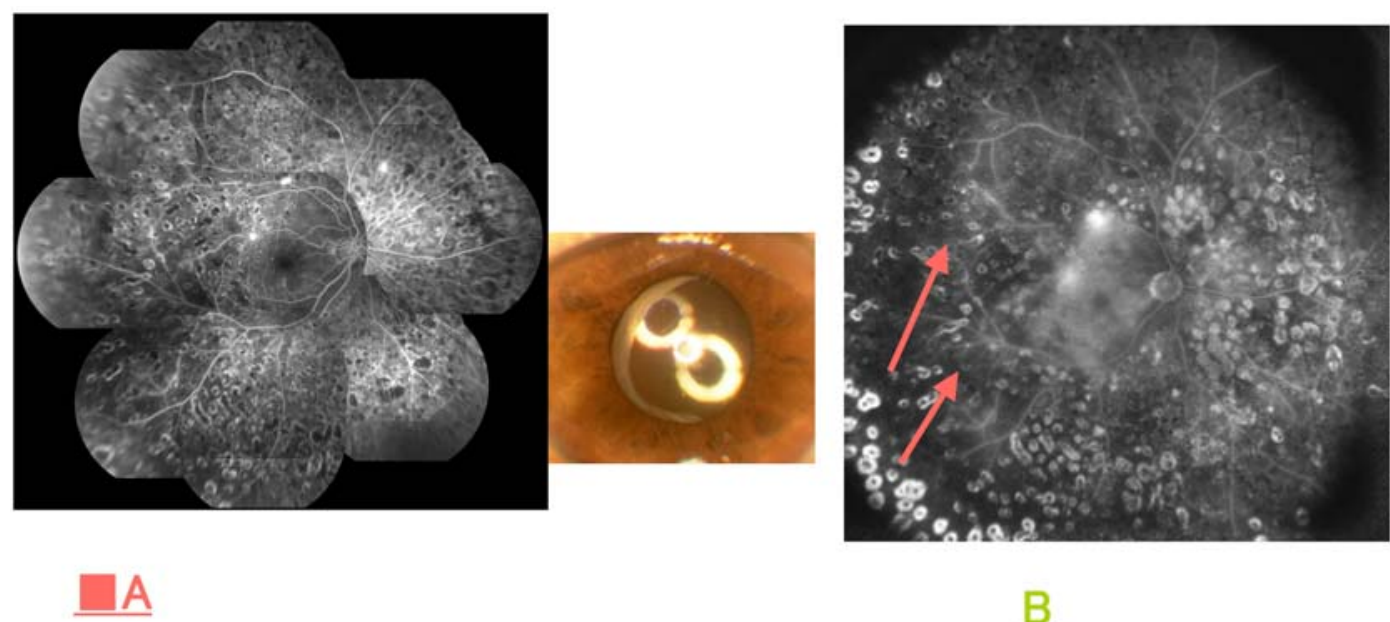

Fig. (6). Case with anterior capsular shrinkage after cataract surgery. A: Ordinary camera; B: HRA2.
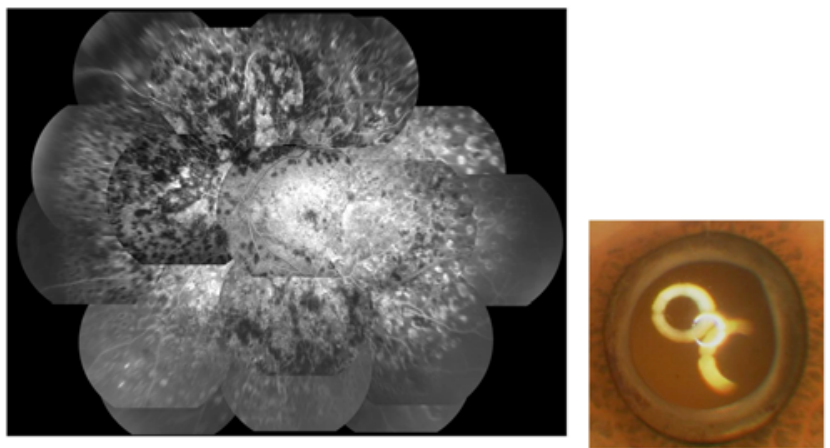

IOL:YA60BBR +24.0D
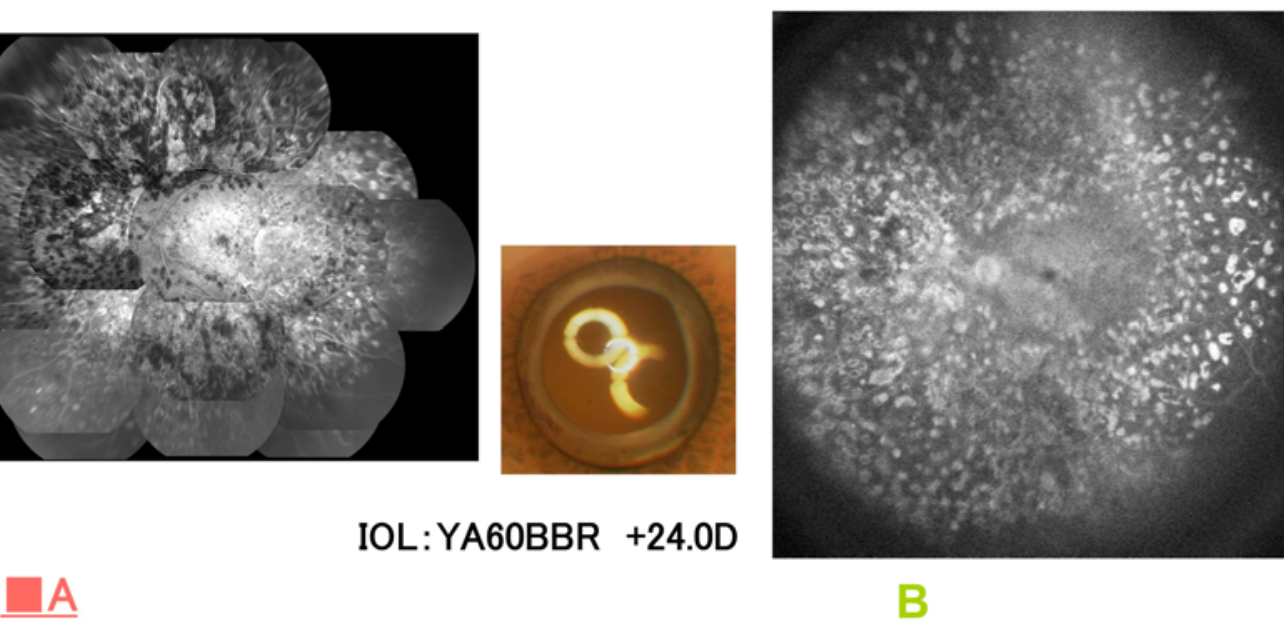

Fig. (7). Case with a yellow IOL. A: HRA2; B: Ordinary camera.

\section{CONFLICT OF INTEREST}

The authors confirm that this article content has no conflict of interest.

\section{ACKNOWLEDGEMENTS}

We have no disclosure, no financial support and no proprietary interest.

\section{REFERENCES}

Holz FG, Bellmann C, Rohrschneider K, et al. Simultaneous confocal laser fluorescein and indocyanine green angiography. Am J Ophthalmol 1998; 125: 227-36.

[2] Hassenstein A, Meyer $\mathrm{CH}$. Clinical use and research applications of Heidelberg retinal angiography and spectral-domein optical coherence tomography-a review. Clin Exp Ophthalmol 2009; 37: $130-43$.
[3] Noyori KS, Chino K, Deguchi T. Wide field fluorescein angiography by use of contact lens. Retina 1983; 3: 131-4.

[4] Staurenghi G, Viola F, Mainster MA, et al. Scanning laser ophthalmoscopy and angiography with a wide-field contact lens system. Arch Ophthalmol 2005; 123: 244-52.

[5] Yamamoto $\mathrm{M}$, Tomii $\mathrm{J}$, Narumi $\mathrm{T}$, et al. Indocyanine green angiography using confocal laser-scanning system and a highresolution wide field lens. Jpn J Clin Opthalmol (in Japanese with English abstract) 2004; 58: 707-10.

[6] Witmer MT, Kiss S. Wide-field imaging of the retina. Surv Ophthalmol 2013; 58: 143-54.

[7] Reeves GM, Kumar N, Beare NA, et al. Use of Ataurenghi len angiography in the management of posterior uvetis. Acta Ophthalmol 2013; 91: 48-51.

[8] Kaines A, Tsui I, Sarraf D, et al. The use of ultra wide field fluorescein angiographyin evaluation and management of uveitis Semin Ophthalmol 2009; 24: 19-24.

[9] Oliver SC, Schwartz SD. Peripheral vessel leakage (PVL): a new angiographic finding in diabetic retinopathy identified with ultra wide-field fluorescein angiography. Semin Ophthalmol 2010; 25: 27-33.

This is an open access article licensed under the terms of the Creative Commons Attribution Non-Commercial License (http://creativecommons.org/licenses/bync/3.0/) which permits unrestricted, non-commercial use, distribution and reproduction in any medium, provided the work is properly cited. 